\title{
Estado da Arte sobre Analítica da Aprendizagem na América Latina
}

\author{
João Batista Carvalho Nunes \\ Programa de Pós-Graduação em Educação / Mestrado Profissional em Computação \\ Aplicada - Universidade Estadual do Ceará \\ Fortaleza, CE - Brasil \\ joao.nunes@uece.br
}

\begin{abstract}
Learning analytics is considered a young and rapidly growing area. The goal of the research that justified this text was to make the state of the art in scientific journals on the use of learning analytics in Latin America. The bibliographic research was employed as a research method in two abstract databases that covered journals of countries in this region: Scielo and Dialnet. Merely two articles were found in Scielo and eight in Dialnet. Only two articles met the research criterion. The results show that there is much to be done in the research field on learning analytics in Latin America.
\end{abstract}

Resumo. A analítica da aprendizagem é considerada uma área jovem e em rápido crescimento. $O$ objetivo da investigação que fundamentou este texto foi realizar o estado da arte em periódicos científicos sobre o uso analítica da aprendizagem na América Latina. Empregou-se, como método, a pesquisa bibliográfica em duas bases de dados de resumos que abrangessem periódicos dos países dessa região: Scielo e Dialnet. Foram encontrados somente dois artigos na Scielo e oito na Dialnet. Desses dez, apenas dois satisfaziam o critério da investigação. Os resultados apontam que há muito a ser feito no campo da pesquisa em analítica da aprendizagem na América Latina.

\section{Introdução}

O acelerado desenvolvimento científico e tecnológico do mundo atual está a exigir dos países maior qualificação de sua força de trabalho. A competitividade das empresas, a necessidade de garantia do emprego e a demanda por melhores serviços públicos pressionam por uma formação de qualidade desde a educação básica até a superior.

Paralelamente, diante da crise econômica mundial, com reflexos em todos os setores da sociedade, a eficiência do sistema educacional é buscada pelos países (OECD, 2012). As instituições de ensino nos países desenvolvidos, como resposta à demanda por maior eficiência, produtividade e prestação de contas de suas ações, estão implementando sistemas de avaliação. Diante disso, os professores são levados a adotar "novas ferramentas e estratégias que lhes permitam identificar rapidamente estudantes em risco e conceber formas de apoiar a sua aprendizagem" (Macfadyen e Dawson, 2010, p. 589).

Por outro lado, observa-se o crescimento exponencial da quantidade de informação armazenada digitalmente no mundo (Hilbert e López, 2011). Continuamente, são produzidos e armazenados dados digitais em servidores, 


\section{CBIE-LACLO 2015}

Anais dos Workshops do IV Congresso Brasileiro de Informática na Educação (CBIE 2015)

computadores de mesa, dispositivos móveis (notebooks, tablets, smartphones etc.) ou unidades de armazenamento portáteis (CD, DVD, pendrive, HD externo etc.).

Diante desse cenário, surgiu a analítica (analytics) como uma solução baseada em tecnologia, que permite coletar e analisar dados, ajudando a "avaliar ações passadas e estimar o potencial de ações futuras, de modo a se tomar melhores decisões e adotar estratégias mais eficazes como organizações ou indivíduos" (Cooper, 2012, p. 3).

Atualmente, vários termos e conceitos relacionados à analítica no campo da educação convivem na literatura científica (Barneveld, Arnold e Campbell, 2012). Ferguson (2013) destaca três expressões que representam áreas de pesquisa com interesses similares e que se sobrepõem: "mineração de dados educacionais" (educational data mining), "analítica acadêmica" (academic analytics) e "analítica da aprendizagem" (learning analytics).

Embora considerada interdisciplinar, a área de mineração de dados educacionais está mais relacionada a pesquisas desenvolvidas na área de Computação. Segundo Romero e Ventura (2013, p. 12), ela "se preocupa com desenvolvimento, pesquisa e aplicação de métodos computadorizados para detectar padrões em grandes conjuntos de dados educacionais [...]". Siemens e Baker (2012) chamam a atenção para as similaridades e distinções entre a mineração de dados educacionais e a analítica da aprendizagem. Para eles, ambos os campos procuram "melhorar a qualidade da análise de dados educacionais em larga escala, a fim apoiar tanto a pesquisa básica como a prática em educação" (p. 253). Por outro lado, uma das principais diferenças entre elas é o fato de que a mineração de dados educacionais enfatiza a redução dos dados a seus componentes e a análise desses componentes individuais e das relações entre eles (abordagem reducionista), enquanto a analítica da aprendizagem busca compreender os sistemas como um todo (abordagem holística).

Campbell e Oblinger $(2007$, p. 3) assinalam que a analítica acadêmica "casa grandes conjuntos de dados, com técnicas estatísticas e modelagem preditiva para melhorar a tomada de decisão", e apresenta "o potencial de aperfeiçoar o ensino, a aprendizagem e o sucesso do aluno". A analítica acadêmica envolve, portanto, procedimentos de coleta, seleção, organização, armazenagem e emissão de relatórios de uma grande quantidade de dados acadêmicos, articulando dados agrupados com técnicas estatísticas e modelos preditivos, que podem ser "usados para informar tanto práticas pedagógicas como políticas" (Macfadyen e Dawson, 2010, p. 590). Ela tem sido usada há alguns anos para subsidiar admissões e a angariação de fundos em várias instituições de ensino superior (Campbell, Deblois e Oblinger, 2007), assim como pode ser empregada para apoiar a tomada de decisões em nível nacional e internacional (Ferguson, 2012).

A analítica da aprendizagem, segundo Johnson, Adams e Cummins (2012, p. 22), "refere-se à interpretação de uma ampla gama de dados reunidos dos estudantes ou produzidos por estes, a fim de avaliar o progresso acadêmico, prever o desempenho futuro e identificar potenciais problemas". Ela é usada para melhorar a aprendizagem e a educação. Para a Society for Learning Analytics Research (SoLAR), a analítica da aprendizagem é a "medição, coleta, análise e divulgação de dados sobre os alunos e seus contextos, com o propósito e compreender e otimizar a aprendizagem e os ambientes em que ela ocorre". 


\section{CBIE-LACLO 2015}

Anais dos Workshops do IV Congresso Brasileiro de Informática na Educação (CBIE 2015)

Para alguns autores a distinção entre as expressões analítica acadêmica e analítica da aprendizagem é mais de terminologia do que conceitual, o que os leva a usá-las como se significassem aproximadamente o mesmo (Mattingly, Rice e Berge, 2012). Existe, contudo, a tentativa de precisar o foco de cada uma dessas áreas. Segundo alguns pesquisadores, o foco da analítica acadêmica são as instituições e sistemas educacionais, enquanto a analítica da aprendizagem centra sua atenção para ajudar estudantes e professores a alcançarem os objetivos de aprendizagem (Barneveld, Arnold e Campbell, 2012; Ferguson, 2013).

Deve-se ressaltar que, segundo o NMC Horizon Report: 2014 Higher Education Edition, cujo propósito é identificar e descrever as tecnologias que terão grande impacto na educação ao redor do mundo nos próximos cinco anos, a analítica da aprendizagem é uma tendência a ser adotada na educação superior em um ano ou menos (Johnson et al., 2014b). Na educação básica, o NMC Horizon Report: 2014 K-12 Edition informa que se trata de tendência a ser absorvida nos próximos dois a três anos (Johnson et al., 2014a). Esse tempo é o mesmo estimado pelo relatório NMC Perspectivas Tecnológicas: Educación Superior en América Latina 2013-2018 para a adoção na educação superior da América Latina (Johnson et al., 2013).

Embora se reconheça a convivência e a partilha de conhecimentos e técnicas entre as comunidades de pesquisadores em mineração de dados educacionais, analítica acadêmica e analítica da aprendizagem, este texto será circunscrito aos estudos e pesquisas voltados à analítica da aprendizagem. $O$ objetivo da investigação que $o$ fundamentou foi realizar o estado da arte em periódicos científicos sobre o uso da analítica da aprendizagem na América Latina.

As investigações sobre a denominação de "estado da arte" podem ser mais abrangentes ou mais restritas quanto às fontes de informação utilizadas. Segundo Romanowski e Ens (2006), uma pesquisa dessa natureza deve compreender o levantamento em distintas fontes de informação, tais como artigos de periódicos, trabalhos em eventos, relatórios de pesquisa, dissertações e teses de uma área de conhecimento. Por outro lado, Nóbrega-Therrien e Therrien (2010, p. 38) afirmam que, no estado da arte, utilizam-se "predominantemente resumos e catálogos de fontes de produção científica".

Quando a investigação se limita a somente um tipo de publicação científica, Romanowski e Ens (2006) afirmam se tratar de um "estado do conhecimento", em vez de um "estado da arte". Outros autores, contudo, consideram não haver distinção entre "estado da arte" e "estado do conhecimento" (Ferreira, 2002; Teixeira, 2006).

As opiniões divergentes sobre a abrangência de um estado da arte não impedem que se entenda que as pesquisas dessa natureza servem para "mapear os estudos publicados e suas temáticas, por quem foram produzidos, com que objetivos e que conteúdos abordam em relação a determinados temas ou área do conhecimento" (Nóbrega-Therrien e Therrien, 2010, p. 37).

No Brasil, Nunes, Nobre e Sampaio (2013, p. 5) desenvolveram pesquisa cujo objetivo foi "analisar a produção científica no Brasil sobre analítica da aprendizagem ou analítica acadêmica, na área de Educação, mais especificamente no campo da educação a distância". Os autores fizeram um levantamento de teses e dissertações defendidas no Brasil e cadastradas no Banco de Teses da Capes (período 1987-2012), artigos publicados em periódicos científicos brasileiros avaliados como Qualis A1 e A2 na área 


\section{CBIE-LACLO 2015}

Anais dos Workshops do IV Congresso Brasileiro de Informática na Educação (CBIE 2015)

de Educação (período 2002-2012) e trabalhos completos aprovados para as reuniões anuais da Associação Nacional de Pós-Graduação e Pesquisa em Educação (ANPEd) e para o Congresso Internacional ABED de Educação a Distância (CIAED) (período 2002-2012). Ao final, não encontraram nenhuma publicação sobre analítica da aprendizagem ou analítica acadêmica, o que denota tratar-se de área com grande potencial de pesquisa no País.

A análise cientométrica e a de conteúdo nos trabalhos e no programa da International Learning Analytics and Knowledge Conference 2013 (LAK 2013), realizadas por Ochoa et al. (2014), identificaram que, dos 143 autores, apenas um era de país da América Latina, mais precisamente do Equador, representando 0,7\% dos autores com participação nesse evento. Os países com o maior número de autores foram Estados Unidos (51 autores), Reino Unido (20 autores), Alemanha (18 autores) e Grécia e Holanda (9 autores cada).

Consulta às bases de dados de resumos multidisciplinares Scopus e Web of Science (julho/2015) não identificou, ademais, a existência de publicações sobre o estado da arte em analítica da aprendizagem no mundo ou limitada à América Latina. Esse achado reforça a justificativa da presente investigação.

Descreve-se, a seguir, o delineamento metodológico utilizado para a realização da pesquisa. Na sequência, os resultados obtidos são explicitados. Como remate, traçam-se as conclusões decorrentes da investigação.

\section{Metodologia}

Para dar resposta ao objetivo da investigação, empregou-se, como método, a pesquisa bibliográfica. Dada a quantidade de periódicos científicos existentes na América Latina, optou-se por utilizar bases de dados de resumos que compreendessem periódicos dos países integrantes dessa região. Para selecionar quais bases de dados seriam utilizadas, foi consultado o Ranking Web of Repositories (http://repositories.webometrics.info/), cujo objetivo é "promover iniciativas de acesso aberto em geral e particularmente de repositórios institucionais" (Aguillo, 2014, p. 125). Usou-se, como critério, o fato de ser iniciativa que congregasse periódicos latino-americanos e estivesse posicionada, no Ranking, entre os dez principais repositórios em julho/2015. Duas bases de dados satisfizeram esse critério: Scielo e Dialnet.

A base Scielo (www.scielo.org) abrange periódicos de 14 países, sendo 11 da América Latina, mais África do Sul, Espanha e Portugal. Possui um acervo de 1.267 periódicos e 561.512 artigos (consulta em 08/07/2015). Começou a funcionar em 1998 no Brasil, após projeto-piloto de um ano. Segundo Packer e Meneghini (2014, p. 16), é a "maior provedora de periódicos indexados pelo Diretório de Periódicos de Acesso Aberto (Directory of Open Access Journals - DOAJ)".

Criada em 2001 pela Universidad de La Rioja, Dialnet permite a busca e recuperação de artigos em periódicos, capítulos de livros, monografias, teses, entre outros documentos da Espanha e de países da América Latina. Seu acervo está constituído de 9.461 periódicos (consulta em 08/07/2015).

Como os artigos possuem, geralmente, resumos em inglês (alguns também possuem títulos e palavras-chave nesse idioma), realizou-se a consulta nas bases Scielo e Dialnet, empregando a expressão exata "learning analytics", compreendendo todo o 
período coberto pelas bases. Preferiu-se utilizar a expressão em inglês para evitar possíveis divergências na tradução para o espanhol e o português. Como exemplo dessas divergências de tradução, no Brasil, por exemplo, Johnson et al. (2014c) traduziram a expressão "learning analytics" como "análise da aprendizagem", enquanto Nunes, Nobre e Sampaio (2013) empregaram a expressão "analítica da aprendizagem", também utilizada neste texto.

$\mathrm{Na}$ consulta empreendida em cada base de dados, ademais, foi preciso selecionar apenas artigos sobre analítica da aprendizagem que representassem estudos e pesquisas realizados na América Latina. Por conseguinte, os resumos dos artigos encontrados e, quando necessário, os textos completos foram lidos para se averiguar se atendiam a esse critério.

Definidos os artigos, procedeu-se à leitura dos respectivos textos completos, a fim de identificar e analisar as categorias que são comuns e divergentes quanto à pesquisa sobre analítica da aprendizagem.

\section{Discussão dos Resultados}

A consulta realizada nas bases de dados de resumos Scielo e Dianet indicou a pequena quantidade de artigos sobre analítica da aprendizagem nos periódicos cobertos por esses repositórios. Conforme se pode depreender do Quadro 1, foram encontrados somente dois artigos na Scielo e oito na Dialnet.

Quadro 1. Resultado para a busca da expressão exata "learning analytics" nas bases Scielo e Dialnet - julho/2015

\begin{tabular}{|c|c|c|c|c|}
\hline Base & Ano & Título & Periódico & $\begin{array}{l}\text { País do } \\
\text { Periódico }\end{array}$ \\
\hline Scielo & 2013 & $\begin{array}{l}\text { Mobile innovations in the } \\
\text { education ecosystem }\end{array}$ & Revista de Ingeniería & Colômbia \\
\hline Scielo & 2014 & $\begin{array}{l}\text { Tendencias en el desarrollo } \\
\text { de las TIC y su impacto en } \\
\text { el campo de la enseñanza }\end{array}$ & $\begin{array}{l}\text { Revista Cubana de } \\
\text { Ciencias Informáticas }\end{array}$ & Cuba \\
\hline Dialnet & 2012 & Gradient, UC3M & $\begin{array}{l}\text { IE Comunicaciones: } \\
\text { Revista Iberoamericana } \\
\text { de Informática } \\
\text { Educativa }\end{array}$ & Espanha \\
\hline Dialnet & 2013 & $\begin{array}{l}\text { Emerging technologies } \\
\text { landscape on education: A } \\
\text { review }\end{array}$ & $\begin{array}{l}\text { International Journal of } \\
\text { Artificial Intelligence } \\
\text { and Interactive } \\
\text { Multimedia }\end{array}$ & Espanha \\
\hline Dialnet & 2014 & $\begin{array}{l}\text { Learning analytics para } \\
\text { predecir la deserción de } \\
\text { estudiantes a distancia }\end{array}$ & Campus Virtuales & Espanha \\
\hline Dialnet & 2014 & $\begin{array}{l}\text { Colaboración y redes } \\
\text { sociales en la enseñanza } \\
\text { universitária }\end{array}$ & $\begin{array}{l}\text { Comunicar: Revista } \\
\text { científica } \\
\text { iberoamericana de }\end{array}$ & Espanha \\
\hline
\end{tabular}


CBIE-LACLO 2015

Anais dos Workshops do IV Congresso Brasileiro de Informática na Educação (CBIE 2015)

\begin{tabular}{|l|l|l|l|l|}
\hline Dialnet & 2014 & $\begin{array}{l}\text { Analítica visual en e- } \\
\text { learning }\end{array}$ & $\begin{array}{l}\text { El profesional de la } \\
\text { información }\end{array}$ & Espanha \\
\hline Dialnet & 2014 & $\begin{array}{l}\text { Am I doing well? } \\
\text { A4Learning as a self- } \\
\text { awareness tool to integrate } \\
\text { in Learning Management } \\
\text { Systems }\end{array}$ & Campus Virtuales & Espanha \\
\hline Dialnet & 2014 & $\begin{array}{l}\text { From place to virtual space: } \\
\text { reconfiguring student } \\
\text { support for distance and e- } \\
\text { learning in the digital age }\end{array}$ & Open Praxis & Noruega \\
\hline Dialnet & 2015 & $\begin{array}{l}\text { Un juego serio para } \\
\text { desarrollar y evaluar la } \\
\text { competencia de trabajo en } \\
\text { equipo }\end{array}$ & $\begin{array}{l}\text { IE Comunicaciones: } \\
\text { Revista Iberoamericana } \\
\text { de Informática } \\
\text { Educativa }\end{array}$ & Espanha \\
\hline
\end{tabular}

Se fosse realizada a mesma busca na base de dados Scopus (julho/2015), alcançar-se-ia a quantidade de 1.337 artigos. Comparativamente, os 10 artigos encontrados nas bases de dados Scielo e Dialnet representam uma razão aproximada de 1:134, ou seja, a cada um artigo sobre analítica da aprendizagem nas bases consultadas, há 134 na Scopus.

A leitura dos resumos e, em alguns casos, dos textos completos dos artigos sobre analítica da aprendizagem possibilitaram se definir quais representavam estudos e pesquisas realizados na América Latina. O Quadro 2 sintetiza a relação desses dois artigos, representando somente 20\% do total de artigos expressos no Quadro 1.

Quadro 2. Artigos de pesquisas na América Latina sobre "learning analytics" nas bases Scielo e Dialnet - julho/2015

\begin{tabular}{|l|l|l|}
\hline Base & Referência & $\begin{array}{l}\text { País Onde } \\
\text { Aconteceu } \\
\text { a Pesquisa }\end{array}$ \\
\hline Scielo & $\begin{array}{l}\text { Pons, N. L., Martínez, E. Y. V. e Sánchez, E. A. A. (2014), } \\
\text { "Tendencias en el desarrollo de las TIC y su impacto en el } \\
\text { campo de la enseñanza", Revista Cubana de Ciencias } \\
\text { Informáticas, 8(1), p. 127-139. }\end{array}$ & Cuba \\
\hline Dialnet & $\begin{array}{l}\text { García-Tinizaray, D., Ordoñez-Briceño, K. e Torres-Diaz, J, } \\
\text { C. (2014), "Learning analytics para predecir la deserción de } \\
\text { estudiantes a distancia", Campus Virtuales, 3 (1), p. 120- } \\
\text { 126. }\end{array}$ & Equador \\
\hline
\end{tabular}

O fato de haver apenas dois artigos sobre o uso da analítica da aprendizagem na América Latina, dentre os 1.267 periódicos presentes na base Scielo e os 9.461 da Dialnet, não significa, contudo, que não existam outras publicações com essa característica, tais como, artigos em outros periódicos, trabalhos em eventos científicos, 


\section{CBIE-LACLO 2015}

Anais dos Workshops do IV Congresso Brasileiro de Informática na Educação (CBIE 2015)

dissertações e teses não cobertos por essas bases. Mesmo assim, acredita-se que essa quantidade ainda seja pequena se comparada à produção nos Estados Unidos, Canadá, Reino Unido e Austrália.

Apenas para ilustrar a possibilidade de se encontrar publicações não abrangidas pelas bases analisadas, tem-se o registro de artigo sobre o uso da analítica da aprendizagem no Brasil (Rigo et al., 2014), assim como trabalho em evento (Nunes, Nobre e Sampaio, 2013) e dissertações de Mestrado (Cambruzzi, 2014; Chaves, 2015).

Analisando-se as ocorrências obtidas, o objetivo do texto de Pons, Martínez e Sánchez (2014,p. 127-128) é "analisar novas tendências no desenvolvimento das Tecnologias de Informação e Comunicação e comprovar a pertinência de seu uso em ambientes universitários a partir da apresentação de diferentes casos de estudo". Para tanto, os autores se restringem a especificar, como tendências, a analítica da aprendizagem, a analítica acadêmica e a mineração de dados educacionais.

Apenas um caso é mostrado. Trata-se de pesquisa levada a cabo na disciplina Engenharia de Software II, da Universidad de las Ciencias Informáticas (UCI), em La Habana, Cuba. Os dados dessa disciplina, obtidos do ambiente virtual de aprendizagem utilizado na Universidade, foram analisados utilizando-se o software de visualização SNAPP e a ferramenta para pré-processamento e análise de grandes volumes de dados Weka.

A análise dos dados permitiu ao docente da disciplina identificar padrões de comportamento, predizer o desempenho e o nível de acesso ao ambiente dos estudantes. Tais achados favoreceram a tomada de decisão mediante a proposição de estratégias pedagógicas, objetivando a melhoria do processo educacional e a aprovação dos estudantes ao final da disciplina.

A pesquisa desenvolvida por García-Tinizaray, Ordoñez-Briceño e Torres-Diaz (2014) pretendia desenvolver um modelo para prever o abandono de estudantes em cursos a distância. Os autores empregaram somente dados do ambiente virtual de aprendizagem de dois cursos - Sistemas Informáticos e Computação e Advocacia e Gestão Ambiental -, da Universidad Técnica Particular de Loja, no Equador. Dados sobre o uso de materiais em papel utilizados nos cursos não foram utilizados na pesquisa.

Para encontrar o modelo preditivo, foram aplicadas duas técnicas de análise multivariada: análise fatorial e regressão logística. Ao final, o modelo relacionou o abandono a três fatores: acesso, fóruns e recursos. Os autores assinalam, contudo, a necessidade de aperfeiçoar o modelo, considerando também variáveis sociodemográficas.

A analítica da aprendizagem é considerada uma área jovem e em rápido crescimento (Siemens, 2012). A primeira conferência internacional sobre a área aconteceu em 2011, em Banff (Canadá), denominada Learning Analytics and Knowledge (LAK). Na América Latina, acontece a primeira edição do Latin American Workshop on Learning Analytics (LALA) em 2015, dentro da programação do IV Congresso Brasileiro de Informática na Educação (CBIE) e X Conferência LatinoAmericana de Objetos e Tecnologias de Aprendizagem (LACLO). Não é de se surpreender, portanto, que os textos analisados datem de 2014. 


\section{CBIE-LACLO 2015}

Anais dos Workshops do IV Congresso Brasileiro de Informática na Educação (CBIE 2015)

Ambos os artigos utilizaram apenas dados provenientes dos ambientes virtuais de aprendizagem das instituições onde a analítica da aprendizagem foi aplicada. Não fizeram uso, por exemplo, de bases de dados sobre características sociodemográficas dos estudantes. Tampouco foram encontradas pesquisas que tratassem do emprego da analítica da aprendizagem no ensino presencial.

As pesquisas foram realizadas em instituições universitárias: uma pública e outra privada. Limitaram-se ao nível da graduação. Não há registros de investigações com foco na educação básica nem em nível de pós-graduação.

$\mathrm{Na}$ análise dos dados, foram empregadas técnicas estatísticas (análise fatorial e regressão logística), de mineração de dados (árvores de decisão) e de visualização baseada em grafos. Somente Pons, Martínez e Sánchez (2014) citam quais softwares foram utilizados na análise dos dados: SNAPP e Weka.

\section{Conclusão}

Os resultados apontam que há muito a ser feito no campo da pesquisa em analítica da aprendizagem na América Latina. O número reduzido de publicações encontradas nas bases analisadas reflete o escasso interesse ainda reinante na comunidade de pesquisadores latino-americanos, mesmo diante das previsões de Johnson et al. (2013).

Faz-se necessário estimular os pesquisadores latino-americanos, incluindo os das áreas de Ciências Humanas, Sociais, Exatas e da Saúde, a constituírem comunidades de pesquisa em torno do interesse em analítica da aprendizagem, de modo a contribuir com sua aplicação em todos os níveis e modalidades da educação, não se restringindo à educação a distância. A analítica da aprendizagem pode ser empregada da educação infantil ao doutorado, desde que se tenham dados confiáveis, acessíveis e no formato digital, para permitir sua análise e a tomada de decisão sobre o processo educacional em curso.

As ocorrências de publicações não contempladas nas bases analisadas indicam a necessidade de estender esta pesquisa, incluindo não apenas artigos nas principais bases de dados de resumos de periódicos da América Latina, mas também os periódicos não abrangidos por essas bases (Journal of Learning Analytics, Revista Brasileira de Informática na Educação etc.), assim como trabalhos em eventos (LAK, TISE, LACLO, SBIE etc.) e dissertações e teses defendidas na região.

\section{Referências}

Aguillo, I. F. (2014), "Análise comparativa dos principais portais ibero-americanos de periódicos científicos: construção de indicadores webométricos para o SciELO", in Packer, A. L. et al. (org), SciELO - 15 anos de acesso aberto [livro eletrônico]: um estudo analítico sobre acesso aberto e comunicação científica, Paris: UNESCO, p. 125-149.

Barneveld, A., Arnold, K. E. e Campbell, J. P. (2012), "Analytics in higher education: establishing a common language", ELI Paper, 1, p. 1-11 [disponível em http://www.educause.edu/ir/library/pdf/ELI3026.pdf, acessado em 20 jan. 2014].

Cambruzzi, W. L. (2014), GVWISE: uma aplicação de learning analytics para a redução da evasão na educação a distância, Dissertação, Mestrado em Computação Aplicada, Universidade do Vale do Rio dos Sinos, São Leopoldo. 


\section{CBIE-LACLO 2015}

Anais dos Workshops do IV Congresso Brasileiro de Informática na Educação (CBIE 2015)

Campbell, J. P., Deblois, P. B. e Oblinger, D. G. (2007), "Academic analytics. a new tool for a new era", EDUCAUSE Review, 42 (4), p. 41-57.

Campbell, J. P. e Oblinger, D. G. (2007), "Academic analytics", Washington: EDUCAUSE Center for Applied Research [disponível em http://wwwcdn.educause.edu/ir/library/pdf/PUB6101.pdf, acessado em 10 jan. 2013].

Chaves, J. B. (2015), Formação a distância de professores em Matemática pela UAB/UECE: relação entre interação e desempenho à luz da analítica da aprendizagem, Dissertação, Mestrado Acadêmico em Educação, Centro de Educação, Universidade Estadual do Ceará, Fortaleza.

Cooper, A. (2012), “A brief history of analytics", CETIS Analytics Series, 1(9), p. 1-21 [disponível em http://publications.cetis.ac.uk/2012/529, acessado em 30 maio 2014].

Ferguson, R. (2012), "Learning analytics: Drivers, developments and challenges", International Journal of Technology Enhanced Learning, 4 (5-6), p. 304-317.

Ferguson, R. (2013), "Learning Analytics for open and distance education", CEMCA EdTech Notes, s/n, p. 1-8.

Ferreira, N. S. A. (2002), "As pesquisas denominadas "estado da arte", Educação \& Sociedade, 79, p. 257-272.

García-Tinizaray, D., Ordoñez-Briceño, K. e Torres-Diaz, J, C. (2014), "Learning analytics para predecir la deserción de estudiantes a distancia”, Campus Virtuales, 3 (1), p. 120-126.

Hilbert, M. e López, P. (2011), “The world's technological capacity to store, communicate, and compute information", Science, 332, p. 60-65.

Johnson, L., Adams Becker, S., Estrada, V. e Freeman, A. (2014a), NMC Horizon Report: 2014 K-12 Edition, Austin, Texas: The New Media Consortium.

Johnson, L., Adams Becker, S., Estrada, V. e Freeman, A. (2014b), NMC Horizon Report: 2014 Higher Education Edition, Austin, Texas: The New Media Consortium.

Johnson, L., Adams Becker, S., Cummins, M. e Estrada, V. (2014c), 2014 NMC Technology Outlook for Brazilian Universities: A Horizon Project Regional Report, Austin, Texas: New Media Consortium.

Johnson, L., Adams Becker, S., Gago, D. Garcia, E. e Martín, S. (2013), NMC Perspectivas Tecnológicas: Educación Superior en América Latina 2013-2018. Un Análisis Regional del Informe Horizon del NMC, Austin, Texas: The New Media Consortium.

Johnson, L., Adams S. e Cummins, M. (2012), The NMC Horizon Report: 2012 Higher Education Edition, Austin, Texas: The New Media Consortium.

Macfadyen, L. P. e Dawson, S. (2010), "Mining LMS data to develop an 'early warning system' for educators: a proof of concept”, Computers \& Education, 54, p. 588-599.

Mattingly, K. D., Rice, M. C. e Berge, Z. L. (2012), "Learning analytics as a tool for closing the assessment loop in higher education", Knowledge Management \& ELearning: An International Journal, 4(3), p. 236-247. 


\section{CBIE-LACLO 2015}

Anais dos Workshops do IV Congresso Brasileiro de Informática na Educação (CBIE 2015)

Nóbrega-Therrien, S. M. e Therrien, J. (2010), "O estado da questão: aportes teóricometodológicos e relatos de sua produção em trabalhos científicos”. In: Farias, I. M. S., Nunes, J.B. C. e Nóbrega-Therrien, S. M. (org.), Pesquisa científica para iniciantes: caminhando no labirinto, Fortaleza: EdUECE, volume 1, p. 33-51.

Nunes, J. B. C., Nobre, J. G. e Sampaio, D. S. (2013), "Retrato da produção científica brasileira sobre analítica da aprendizagem: potencial para a educação a distância", Anais do XXI Encontro de Pesquisa Educacional do Norte e Nordeste (EPENN), Recife: UFPE, p. 1-17.

Ochoa, X., Suthers, D., Verbert, K. e Duval, E. (2014), "Analysis and Reflections on the Third Learning Analytics and Knowledge Conference (LAK 2013)", Journal of Learning Analytics, 1(2), p. 5-22.

OECD (2012), Education at a glance 2012: OECD indicators. S.l.: OECD Publishing, 2012 [disponível em http://dx.doi.org/10.1787/eag-2012-en, acessado em 20 jan. 2013].

Packer, A. L. e Meneghini, R. (2014), "SciELO aos 15 anos: raison d'être, avanços e desafios para o futuro". in Packer, A. L. et al. (org.), SciELO - 15 anos de acesso aberto [livro eletrônico]: um estudo analítico sobre acesso aberto e comunicação científica, Paris: UNESCO, p. 15-28.

Pons, N. L., Martínez, E. Y. V. e Sánchez, E. A. A. (2014), “Tendencias en el desarrollo de las TIC y su impacto en el campo de la enseñanza", Revista Cubana de Ciencias Informáticas, 8(1), p. 127-139.

Rigo, S. J., Cambruzzi, W., Barbosa, J. L. V. e Cazella, S. C. (2014), “Aplicações de mineração de dados educacionais e learning analytics com foco na evasão escolar: oportunidades e desafios", Revista Brasileira de Informática na Educação, 22(1), p. 132-146.

Romanowski, J. P. e Ens, R. T. (2006), “As pesquisas denominadas do tipo 'estado da arte' em educação", Revista Diálogo Educacional, 6(19), p. 37-50.

Romero, C. e Ventura, S. (2013), "Data mining in education", WIREs Data Mining Knowl Discov, 3, p. 12-27.

Siemens, G. (2012), "Learning analytics: envisioning a research discipline and a domain of practice". Proceedings of the 2nd International Conference on Learning Analytics and Knowledge, New York: ACM, p. 4-8.

Siemens, G. e Baker, R. S. J. D. (2012), "Learning analytics and educational data mining: towards communication and collaboration". Proceedings of the 2nd International Conference on Learning Analytics and Knowledge, New York: ACM, p. 252-254.

Teixeira, C. R. (2006), “O 'estado da arte': a concepção de avaliação educacional veiculada na produção acadêmica do Programa de Pós-Graduação em Educação: Currículo (1975-2000)", Cadernos de Pós-Graduação - educação, 5(1), p. 59-66. 